\title{
Albumin-Globulin Ratio Is an Independent Determinant of 28-Day Mortality in Patients with Critical Illness
}

\author{
Bin Liu, ${ }^{1,2}$ Kun Xiao, ${ }^{1}$ Peng Yan, ${ }^{1}$ Tianyu Sun, ${ }^{1}$ Jiang Wang, ${ }^{1,2}$ Fei Xie, ${ }^{1}$ Guoxin Mo ${ }^{1},{ }^{1}$ \\ and Lixin Xie $\mathbb{1}^{1}$ \\ ${ }^{1}$ College of Pulmonary and Critical Care Medicine, Chinese PLA General Hospital, China \\ ${ }^{2}$ Medical School of Chinese PLA, China \\ Correspondence should be addressed to Guoxin Mo; 13661005996@139.com and Lixin Xie; xielx301@126.com
}

Received 11 March 2021; Revised 8 June 2021; Accepted 15 July 2021; Published 26 August 2021

Academic Editor: Hubertus Himmerich

Copyright ( 2021 Bin Liu et al. This is an open access article distributed under the Creative Commons Attribution License, which permits unrestricted use, distribution, and reproduction in any medium, provided the original work is properly cited.

\begin{abstract}
Background. Critical illness in the intensive care unit (ICU) has been a global health priority. Systemic nutritional status has turned out to be related to the prognosis of critically ill patients. The albumin-globulin ratio (AGR) has been reported to be a novel prognostic factor of many diseases. This study is aimed at investigating whether the AGR could predict the mortality risk in critically ill patients. Methods. We enrolled 582 adult patients admitted to the respiratory intensive care unit (RICU). We collected the clinical and laboratory data. X-tile software was used to determine the optimal cut-off values for the AGR. Patients were divided into three groups according to the AGR (low AGR group with AGR $<0.8$, medium AGR group with AGR ranging from 0.8 to 1.1, and high AGR group with AGR > 1.1). Kaplan-Meier analysis was used for survival analysis. A Cox proportional hazard model was applied to the univariate and multivariate analyses for the potential predictors associated with survival. Results. Our present study showed that the AGR was related to the 28-day survival of critically ill patients in the RICU. The rate of pneumonia in the low AGR group was significantly higher than that in the other groups. Patients with a lower AGR present an increased risk of 28-day mortality compared to patients with a higher AGR. Cox regression analysis showed that the AGR might be an independent predictor of prognosis to 28-day survival in critically ill patients in the RICU. Medium and high AGR values remained independently associated with better 28-day survival than low AGR values (HR: 0.484 (0.263-0.892) $(p=0.02)$; HR: $0.332(0.166-0.665)(p=0.002))$. Conclusion. The AGR might be an independent predictor of prognosis in critically ill patients.
\end{abstract}

\section{Introduction}

Critical illnesses in the intensive care unit (ICU), such as sepsis, could be a global health priority $[1,2]$. Being bedridden and the use of some drugs may affect the nutritional status of critically ill patients. Some studies have shown that systemic nutritional status is related to the prognosis of critically ill patients [3].

Serum albumin is commonly used as a surrogate of nutritional status, and serum globulin is cited to assess the severity of chronic inflammation [4]. Hypoalbuminemia has turned out to be associated with critical illness through multiple mechanisms [5]. The albumin-globulin ratio (AGR), calculated as serum albumin/(serum protein-serum albumin), has been reported to be a novel prognosticator of many diseases, such as lung cancer and microscopic polyangiitis [6, 7]. However, previous studies have mainly focused on patients with cancer and some chronic diseases, and thus far, no study has investigated the association of the AGR with the prognosis of critically ill patients. We aimed to conduct this study to determine whether the AGR would predict survival in patients with critical illness.

\section{Materials and Methods}

2.1. Participants and Study Design. We conducted a retrospective cohort study of 582 adult patients admitted to the respiratory intensive care unit (RICU) in the College of 
TABLE 1: The clinical characteristics of patients admitted to RICU by AGR.

\begin{tabular}{|c|c|c|c|c|c|}
\hline Variable & Total & Low AGR $(\leq 0.8)$ & $\begin{array}{c}\text { Medium AGR } \\
(0.8-1.1) \\
\end{array}$ & High AGR $(>0.8)$ & $p$ value \\
\hline \multicolumn{6}{|l|}{ Demographics } \\
\hline Gender (female, $n, \%$ ) & $198(34.0 \%)$ & $26(27.7 \%)$ & $86(36.3 \%)$ & $86(34.3 \%)$ & 0.319 \\
\hline Age (y) & 62 & 61 & 63 & 61 & 0.196 \\
\hline \multicolumn{6}{|l|}{ Comorbidity } \\
\hline Pneumonia $(n, \%)$ & $267(45.9 \%)$ & $58(61.7 \%)$ & $114(48.1 \%)$ & $95(37.8 \%)$ & $<0.001$ \\
\hline Respiratory failure (n,\%) & $93(15.8 \%)$ & $16(17.0 \%)$ & $44(18.6 \%)$ & $33(13.1 \%)$ & 0.273 \\
\hline \multicolumn{6}{|l|}{ Laboratory variables } \\
\hline ALT ave (U/L) & 34 & 34 & 38 & 29 & 0.107 \\
\hline AST ave (U/L) & 37 & 52 & 39 & 30 & 0.047 \\
\hline DBIL ave $(\mu \mathrm{mol} / \mathrm{L})$ & 6.8 & 6.6 & 7 & 6.6 & 0.967 \\
\hline TBIL ave $(\mu \mathrm{mol} / \mathrm{L})$ & 14.8 & 13.5 & 15.1 & 14.9 & 0.828 \\
\hline \multicolumn{6}{|l|}{ Patient outcomes } \\
\hline 28-day mortality & $10.0 \%$ & $19.1 \%$ & $10.5 \%$ & $6.0 \%$ & 0.001 \\
\hline Length of stay days & 16 & 17 & 17 & 15 & 0.214 \\
\hline
\end{tabular}

Pulmonary and Critical Care Medicine, Chinese PLA General Hospital, from 1 July 2008 to 31 December 2017. We collected the clinical data of these patients for retrospective analysis. The inclusion criteria of the study were as follows: (1) adult patients (age $\geq 18$ years old), (2) patients with a laboratory examination of serum albumin and serum total protein, (3) patients with a laboratory examination of liver function, and (4) patients with a RICU stay of 2 to 100 days. The exclusion criteria of the study were as follows: (1) patients younger than 18 years old and (2) patients with insufficient AGR data. The primary outcome of the study was 28-day mortality.

2.2. Clinical and Laboratory Data Collection. The baseline data of the patients included were collected during the stay in the RICU. The AGR was calculated by the following formula: $\quad \mathrm{AGR}=$ serum albumin $/($ serum protein - serum albumin) [8].

2.3. Statistical Analysis. X-tile 3.6.1 software (Yale University, New Haven, CT, USA) was used to determine the optimal cut-off values for the AGR. IBM SPSS Statistics 21.0 software (SPSS Inc., Chicago, IL, USA) was applied to complete the statistical analysis. Data are described as numbers (percentages) for categorical variables, means \pm standard deviations for normally distributed variables, and medians (interquartile ranges) for skewed distributed variables. Patients were divided into three groups according to the AGR. $X^{2}$ tests were used to analyze the relationship between the clinical parameters and AGR. Kaplan-Meier analysis was used for survival analysis. A Cox proportional hazard model was applied to the univariate and multivariate analyses for the potential predictors associated with survival. The results were considered statistically significant with a two-sided $p$ value $<0.05$.

\section{Results}

3.1. Clinical Characteristics of Patients. The cohort included 198 females and 384 males, with an average age of 62 years old (range 18-98 years old). The AGR of these patients ranged from 0.2 to 2.24 , with a median value of 1.06 . Data on liver function-related factors, such as alanine aminotransferase (ALT), aspartate aminotransferase (AST), total bilirubin (TBIL), and direct bilirubin (DBIL), were collected. The average ALT, AST, TBIL, and DBIL levels were $34 \mathrm{U} / \mathrm{L}$, $37 \mathrm{U} / \mathrm{L}, 14.8 \mu \mathrm{mol} / \mathrm{L}$, and $6.8 \mu \mathrm{mol} / \mathrm{L}$, respectively. Among these 582 patients, $267(45.9 \%)$ patients had a comorbidity of pneumonia, and $93(15.8 \%)$ patients had a comorbidity of respiratory failure. The average length of stay (LOS) in the RICU was 16 days. The 28-day mortality for the entire cohort was $10.0 \%$ (Table 1 ).

3.2. Identification of the Optimal Cut-off Value for AGR. We used $X$-tile software to determine the optimal cut-off value for the AGR of 28-day mortality. $X$-tile is a robust graphical tool verified by Yale University [9], and the cut-off value of the AGR by $X$-tile could be convincing. The optimal cut-off values determined by $X$-tile for the AGR were 0.8 and 1.1 (Figure 1). According to the cut-off value, we divided the patients into three groups: low AGR group with $\mathrm{AGR}<0.8$, medium AGR group with AGR ranging from 0.8 to 1.1, and high AGR group with AGR > 1.1. Ninety-four patients were in the low AGR group, 237 patients were in the medium AGR group, and 251 patients were in the high AGR group. For the patients with pneumonia, there were $58(61.7 \%)$, $114(48.1 \%)$, and $95(37.8 \%)$ patients in the low AGR, medium AGR, and high AGR groups, respectively $(p<0.001)$ (Table 1$)$. The rate of patients with pneumonia in the low AGR group was significantly higher than that in the other groups. The 28-day mortality of the low AGR, 


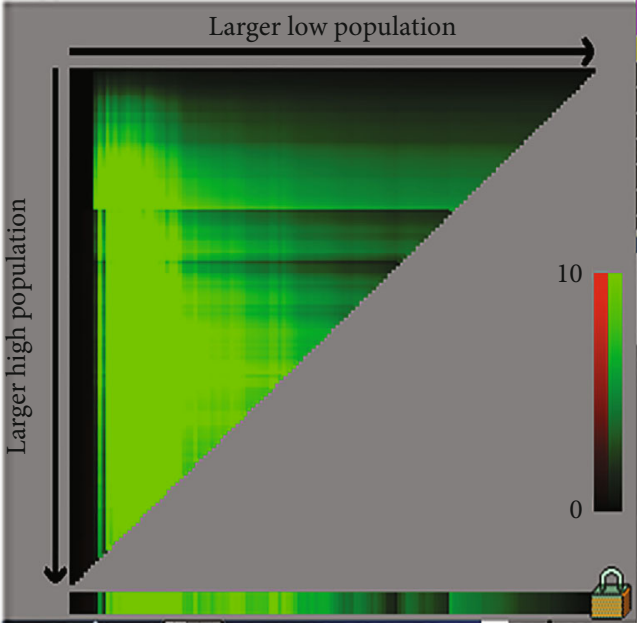

(a)

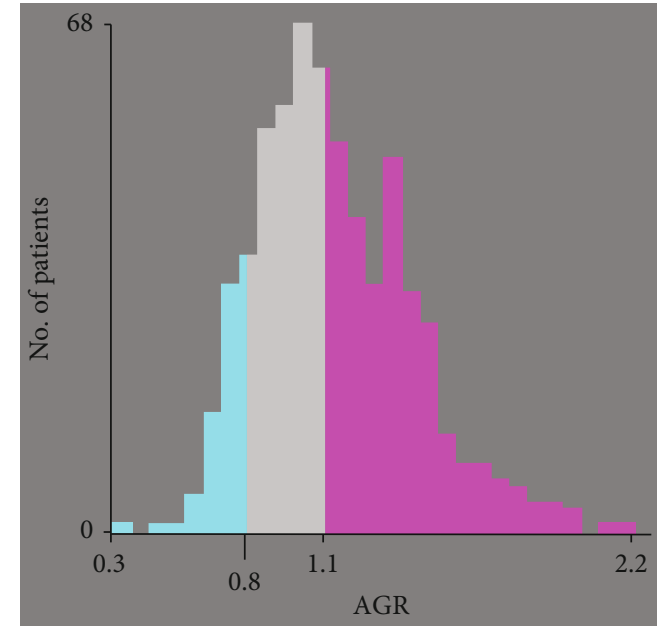

(b)

FIGURE 1: $X$-tile analyses of 28-day mortality performed by using patients' data to determine the optimal cut-off values for $D$-dimer. The sample of critically ill patients was equally divided into training and validation sets. $X$-tile plots of training sets are shown in (a), with plots of matched validation sets shown in the smaller inset (a). The optimal cut-off value highlighted by the black circles in (a) is shown in histograms of the entire cohort (b).

medium AGR, and high AGR groups was 19.1\%, 10.5\%, and $6.0 \%$, respectively $(p=0.001)$.

3.3. Kaplan-Meier Analysis. Figure 2 illustrates the KaplanMeier analysis of 28-day survival with $\log -\operatorname{rank} p=0.004$. Kaplan-Meier analysis showed that the low AGR group had the worst 28-day survival, and the high AGR group had the best 28-day survival, indicating that the AGR might be related to the prognosis of critically ill patients in the RICU.

\subsection{Univariate and Multivariate Analyses of Predictive} Factors for 28-Day Survival. Cox proportional hazard regression analysis was conducted to determine the potential prognostic factors for the 28-day survival of critically ill patients in the RICU. The univariate analysis results showed that the medium AGR and high AGR groups had better 28-day survival than the low AGR group, with hazard ratios (HRs) of $0.523(0.285-0.959)(p=0.036)$ and $0.330(0.166-0.655)$ $(p=0.002)$, respectively. Meanwhile, elevated DBIL and TBIL and age $\geq 65$ years old indicated a correlation with worse 28-day survival (HR: $2.232(1.306-3.815)(p=0.003)$, HR: $2.016(1.156-3.517)(p=0.014)$, and HR: $2.19(1.231-$ 3.896) $(p=0.008)$ ), respectively). In the multivariate analysis, medium and high AGR values remained independently associated with better 28-day survival (HR: $0.484(0.263-0.892)$ $(p=0.02)$ and HR: $0.332(0.166-0.665)(p=0.002))$ than low AGR values. Age exceeding 65 years remained an independent factor for worse 28-day survival (HR: 2.196 (1.233.92) $(p=0.009))($ Table 2).

\section{Discussion}

To the best of our knowledge, this is the first study to examine the prognostic value of the AGR in terms of 28-day survival in critically ill patients. Our present study showed

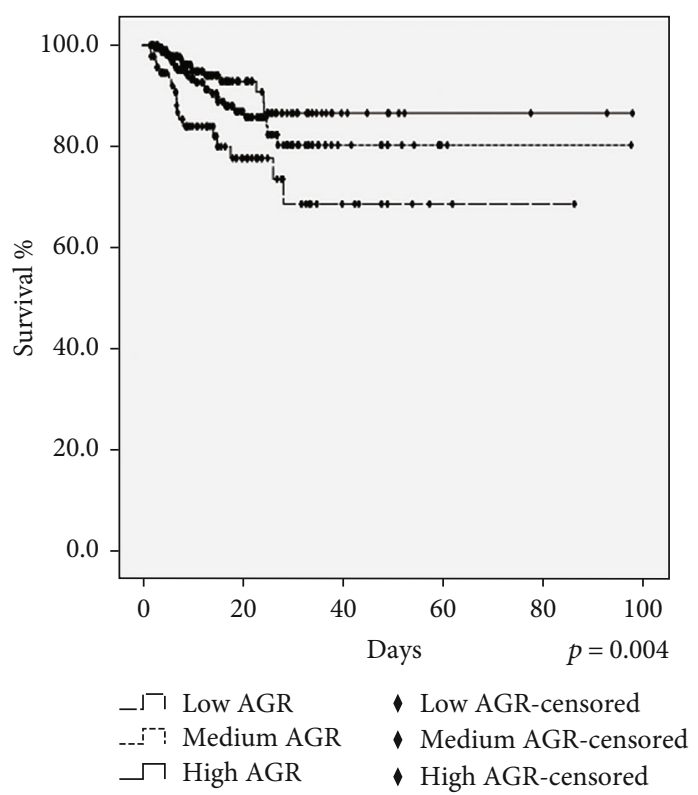

FIgURe 2: Kaplan-Meier analysis showed that the low AGR group had the worst 28-day survival, and the high AGR group had the best 28-day survival.

that the AGR was related to the 28-day survival of critically ill patients in the RICU. The rate of pneumonia in the low AGR group was significantly higher than that in the other groups. Patients with a lower AGR present an increased risk of 28-day mortality compared to patients with a higher AGR. Cox regression analysis showed that the AGR might be an independent predictor of prognosis to 28-day survival in critically ill patients in the RICU.

The condition of patients in the ICU is usually poor, causing a serious economic burden to society and family. 
TABLE 2: Univariate and multivariate analysis of prognostic factors for 28-day survival in critically ill patients.

\begin{tabular}{|c|c|c|c|c|}
\hline \multirow{2}{*}{ Variable } & \multicolumn{2}{|c|}{ Univariate analysis } & \multicolumn{2}{|c|}{ Multivariate analysis } \\
\hline & HR (95\% CI) & $p$ value & HR (95\% CI) & $p$ value \\
\hline \multicolumn{5}{|l|}{ Low AGR } \\
\hline Medium AGR & $0.523(0.285-0.959)$ & 0.036 & $0.484(0.263-0.892)$ & 0.02 \\
\hline High AGR & $0.330(0.166-0.655)$ & 0.002 & $0.332(0.166-0.665)$ & 0.002 \\
\hline Age $(\geq 65$ y vs. $<65$ y) & $2.19(1.231-3.896)$ & 0.008 & $2.196(1.23-3.92)$ & 0.009 \\
\hline Gender (female vs. male) & $0.839(0.481-1.464)$ & NS & & \\
\hline AST (elevated vs. normal) & $1.408(0.799-2.481)$ & NS & & \\
\hline ALT (elevated vs. normal) & $1.202(0.659-2.194)$ & NS & & \\
\hline DBIL (elevated vs. normal) & $2.232(1.306-3.815)$ & 0.003 & $1.65(0.734-3.711)$ & NS \\
\hline TBIL (elevated vs. normal) & $2.016(1.156-3.517)$ & 0.014 & $1.513(0.648-3.529)$ & NS \\
\hline
\end{tabular}

Abbreviation: AGR: Albumin-to-globulin ratio; HR: hazard ratio; AST: aspartate aminotransferase; ALT: alanine aminotransferase; DBIL:direct bilirubin; TBIL: total bilirubin; NS: not significant.

Therefore, it is very important to find novel markers in the prognosis of critical illness, which might be helpful in targeted therapy. Agnello et al. showed that monocyte distribution width was associated with sepsis in the ICU [10]. In addition, midregional proadrenomedullin (MR-proADM) has also shown a predictive role in the mortality of critically ill patients in the ICU [11].

The nutritional status of critically ill patients will be affected due to long-term bed stays and inflammation. Therefore, nutritional status was associated with the prognosis of critically ill patients. Nutritional risk has been proven to be a predictor of 28-day and ICU mortality [12]. Many studies have focused on nutrition status and its predictable role in critically ill patients, as well as nutrition therapy $[13,14]$. Albumin is commonly regarded as a biological marker for assessing nutritional status. Critical illness can change the distribution of intravascular and extravascular albumin. In addition, the rate of albumin synthesis and degradation will also be affected, and cytokines, such as tumor necrosis factor- $\alpha$ (TNF- $\alpha$ ) and interleukin-6 (IL-6), turned out to be responsible for the altered albumin in critically ill patients [5]. The etiologies of hypoalbuminemia include malnourishment, hepatic impairment, and decreased hepatic synthesis of albumin [4].

The AGR has drawn increasing attention for its potential role as a predictor in many diseases. The AGR was assumed to be correlated with prognosis in colorectal cancer [15], lung cancer [16], gliomas [17], and renal cell carcinoma [18]. On the one hand, the AGR reflects the nutritional status of the patients, which might indicate the prognosis of the disease. On the other hand, the AGR might be associated with inflammation. Albumin is related to many inflammatory markers, such as C-reactive protein (CRP) [19], interleukin-1(IL-1), IL-6, and TNF- $\alpha$ [4]. Undurti proposed that albumin mobilizes polyunsaturated fatty acids (PUFAs) from the liver and aids in the formation of cytoprotective bioactive lipids, such as lipoxins (LXs), resolvins, and protectins that may underlie its benefits [20]. In our present study, the rate of patients with pneumonia in the low AGR group was significantly higher than that in the other groups, which might indicate that the AGR correlated with pneumonia in critically ill patients. The mechanism of the AGR and critically ill diseases, such as pneumonia, needs to be researched in the future.

Our study showed that the AGR might be an independent predictor of the prognosis to 28-day survival in critically ill patients. A lower AGR indicated a worse prognosis in critically ill patients. We might find an efficient way to increase albumin and the AGR; so, we could give critically ill patients an encouraging prognosis.

\section{Limitations}

There are some limitations to our retrospective study. First, this is a single-centre retrospective study, and the sample size is relatively small compared to that in a multicentre study. In addition, there might be selection bias, which is the defect in most single-centre retrospective studies. Second, we did not collect the AGR during the whole stay in the RICU, and we cannot accurately analyze the trend of the AGR with disease progression. Finally, our study was a clinical study and did not examine the exact mechanism by which the AGR affects the prognosis of critically ill patients. More studies of the mechanisms of the AGR and disease are needed in the future.

\section{Conclusions}

In the present study, we showed that the AGR was correlated with the 28-day survival of critically ill patients in the RICU. Patients with a lower AGR present an increased risk of 28day mortality compared to patients with a higher AGR. AGR might be an independent predictor of prognosis to 28-day survival in critically ill patients. Multi-institutional studies are needed to confirm our present findings. How to adjust the therapeutic strategies of critically ill patients with a poor prognosis determined by the AGR might be a subject worth studying in the future.

\section{Data Availability}

The data used to support the findings of this study are available from the corresponding author upon request. 


\section{Ethical Approval}

This cohort study was approved by the Ethics Committee of Chinese PLA General Hospital. A waiver of informed consent was granted because of minimal risk exposure to patients.

\section{Conflicts of Interest}

The authors declare no conflict of interest.

\section{Authors' Contributions}

$\mathrm{BL}$ and KX are the guarantors of the paper and realized statistics. LXX and GXM designed the study. FX, TYS, and PY ensured the inclusion and follow-up of patients. JW, BL, and KX managed imaging procedures. FX revised the English language. BL and KX analyzed patient records. BL and TSY analyzed the data. All authors contributed to the article and approved the submitted version. Bin Liu and Kun Xiao contributed equally to this work. Guoxin Mo and Lixin Xie are the co-corresponding authors.

\section{References}

[1] R. Markwart, H. Saito, T. Harder et al., "Epidemiology and burden of sepsis acquired in hospitals and intensive care units: a systematic review and meta-analysis," Intensive Care Medicine, vol. 46, no. 8, pp. 1536-1551, 2020.

[2] K. Reinhart, R. Daniels, N. Kissoon, F. R. Machado, R. D. Schachter, and S. Finfer, "Recognizing sepsis as a global health priority - a WHO resolution," The New England Journal of Medicine, vol. 377, no. 5, pp. 414-417, 2017.

[3] P. Pickkers, N. de Keizer, J. Dusseljee, D. Weerheijm, J. G. van der Hoeven, and N. Peek, "Body mass index is associated with hospital mortality in critically ill patients," Critical Care Medicine, vol. 41, no. 8, pp. 1878-1883, 2013.

[4] P.-P. Wu, Y.-P. Hsieh, C.-T. Kor, and P.-F. Chiu, “Association between albumin-globulin ratio and mortality in patients with chronic kidney disease," Journal of clinical medicine, vol. 8, 2019.

[5] V. Gounden, R. Vashisht, and I. Jialal, Hypoalbuminemia, Treasure Island (FL), 2021.

[6] H. Zhang, B. Zhang, K. Zhu et al., "Preoperative albumin-toglobulin ratio predicts survival in patients with non-smallcell lung cancer after surgery," Journal of Cellular Physiology, vol. 234, pp. 2471-2479, 2018.

[7] S. S. Ahn, J. Yoo, S. M. Jung, J. J. Song, Y. B. Park, and S. W. Lee, "Clinical role of albumin to globulin ratio in microscopic polyangiitis: a retrospective monocentric study," Clinical Rheumatology, vol. 38, no. 2, pp. 487-494, 2019.

[8] F. Peng, L. Sun, T. Chen et al., "Albumin-globulin ratio and mortality in patients on peritoneal dialysis: a retrospective study," BMC nephrology, vol. 21, p. 51, 2020.

[9] R. L. Camp, M. Dolled-Filhart, and D. L. Rimm, "X-tile: a new bio-informatics tool for biomarker assessment and outcomebased cut-point optimization," Clinical cancer research, vol. 10, pp. 7252-7259, 2004.

[10] L. Agnello, B. L. Sasso, R. V. Giglio et al., "Monocyte distribution width as a biomarker of sepsis in the intensive care unit: a pilot study," Annals of Clinical Biochemistry, vol. 58, no. 1, pp. 70-72, 2021.

[11] C. Bellia, L. Agnello, B. Lo Sasso et al., "Mid-regional proadrenomedullin predicts poor outcome in non-selected patients admitted to an intensive care unit," Clinical Chemistry and Laboratory Medicine, vol. 57, no. 4, pp. 549-555, 2019.

[12] A. Cattani, I. C. Eckert, J. E. Brito, R. F. Tartari, and F. M. Silva, "Nutritional risk in critically ill patients: how it is assessed, its prevalence and prognostic value: a systematic review," Nutrition reviews, vol. 78, no. 12, pp. 1052-1068, 2020.

[13] P. Singer, E. De Waele, C. Sanchez et al., "TICACOS international: a multi-center, randomized, prospective controlled study comparing tight calorie control versus Liberal calorie administration study," Clinical nutrition, vol. 40, no. 2, pp. 380-387, 2020.

[14] K. Fetterplace, E. J. Ridley, L. Beach et al., "Quantifying response to nutritional therapy during critical illness: implications for clinical practice and research? A narrative review," Journal of parenteral and enteral nutrition, vol. 45, no. 2, pp. 251-266, 2020.

[15] Z. Hu, Y. Li, W. Mao, B. Chen, L. Yang, and X. Meng, "Impact of nutritional indices on the survival outcomes of patients with colorectal cancer," Cancer management and research, vol. 12, pp. 2279-2289, 2020.

[16] J. Li, Y. Wang, Y. Wu, J. Li, and G. Che, "Prognostic value of pretreatment albumin to globulin ratio in lung cancer: a meta-analysis," Nutrition and cancer, vol. 73, no. 1, pp. 7582, 2020.

[17] Z.-Y. Zhang, Y.-B. Zhan, F.-J. Zhang et al., "Prognostic value of preoperative hematological markers combined with molecular pathology in patients with diffuse gliomas," Aging, vol. 11, pp. 6252-6272, 2019.

[18] M. Y. Koparal, F. Polat, S. Çetin, E. C. Bulut, and T. S. Sözen, "Prognostic role of preoperative albumin to globulin ratio in predicting survival of clear cell renal cell carcinoma," International braz j urol, vol. 44, pp. 933-946, 2018.

[19] M. D. Barber, J. A. Ross, and K. C. H. Fearon, "Changes in nutritional, functional, and inflammatory markers in advanced pancreatic cancer," Nutrition and Cancer, vol. 35, pp. 106-110, 1999.

[20] U. N. Das, "Albumin to globulin ratio and/or plasma albumin in predicting long-term mortality," American Journal of Surgery, vol. 208, pp. 157-158, 2014. 